\title{
Rigidity in topological dynamics
}

\author{
S. GLASNER AND D. MAON \\ School of Mathematical Sciences, Tel-Aviv University, Tel-Aviv 69978, Israel
}

(Received 3 March 1987)

Abstract. By analogy with the ergodic theoretical notion, we introduce notions of rigidity for a minimal flow $(X, T)$ according to the various ways a sequence $T^{n_{i}}$ can tend to the identity transformation. The main results obtained are:

(i) On a rigid flow there exists a $T$-invariant, symmetric, closed relation $\tilde{N}$ such that $(X, T)$ is uniformly rigid iff $\tilde{N}=\Delta$, the diagonal relation.

(ii) For syndetically distal (hence distal) flows rigidity is equivalent to uniform rigidity.

(iii) We construct a family of rigid flows which includes Körner's example, in which $\tilde{N}$ exhibits various kinds of behaviour, e.g. $\tilde{N}$ need not be an equivalence relation.

(iv) The structure of flows in the above mentioned family is investigated. It is shown that these flows are almost automorphic.

\section{Introduction}

The notion of (measure theoretical) rigidity was introduced by $\mathrm{H}$. Furstenberg and B. Weiss in [FW]. They call the finite measure preserving system $(X, B, \mu, T)$, rigid if for some sequence $n_{i} \rightarrow \infty$ and every $f \in L_{2}(\mu), T^{n_{i}} f \rightarrow f$ in $L_{2}(\mu)$. B. Weiss has shown that if $T$ is rigid then there exists a subsequence $n_{i}^{\prime}$ of $n_{i}$ and a subalgebra $A \subset L_{\infty}(\mu)$ which is dense in $L_{2}(\mu)$ and such that $\left\|T^{n} f-f\right\|_{\infty} \rightarrow 0$ for every $f \in A$, (private communication).

In this paper we are concerned with analogous notions of rigidity in the setting of topological dynamics and their interrelation. To be specific let $X$ be a metric compact space and $T: X \rightarrow X$ a self homeomorphism. We call the pair $(X, T)$ a flow, and say that

(i) $(X, T)$ is weakly rigid if for every $\varepsilon>0$ and points $x_{1} \cdots x_{n} \in X$, there exists $k \in \mathbb{Z} \backslash\{0\}$ such that $d\left(T^{k} x_{i}, x_{i}\right)<\varepsilon,(i=1,2, \ldots, n)$.

(ii) $(X, T)$ is rigid (with respect to a sequence $n_{k} \nearrow \infty$ ) if $T^{n_{k}} x \rightarrow x \forall x \in X$.

(iii) $(X, T)$ is uniformly rigid (w.r.t. $\left.n_{k}\right)$ if $\lim T^{n_{k}}=$ Identity uniformly on $X$.

Clearly (iii) $\Rightarrow($ ii $) \Rightarrow$ (i). It is easy to construct an example of a rigid flow which is not uniformly rigid. (Take $X=\left\{r e^{i \theta}: 0 \leq \theta \leq 2 \pi, r=1-2^{-n}, n=1,2,3, \ldots\right.$ or $r=1\}$ and $T z=z \exp \left(2 \pi i \cdot 2^{-n}\right)$ when $|z|=1-2^{-n}$ and $T z=z$ if $\left.|z|=1 ; n_{k}=2^{k}\right)$. The question whether (ii) $\Rightarrow$ (iii) becomes more difficult if we require $(X, T)$ to be minimal. The negative answer to this question was given by T. W. Körner who produced an example of a minimal flow, rigid with respect to some sequence $\left\{n_{k}\right\}$ and not uniformly rigid with respect to any sequence including $\left\{n_{k}\right\},[K]$. 
Let $(X, T)$ be a minimal flow, rigid w.r.t. a sequence $\left\{n_{k}\right\}$. Define

$$
\tilde{N}=\left\{\left(x, x^{\prime}\right): \begin{array}{c}
\text { There exists a subsequence } n_{k}^{\prime} \text { of } n_{k} \\
\text { and sequences } x_{k} \rightarrow x \text { and } x_{k}^{\prime} \rightarrow x^{\prime} \\
\text { such that } d\left(T^{n_{k}^{\prime}} x_{k}, T^{n_{k}^{\prime}} x_{k}^{\prime}\right) \rightarrow 0
\end{array}\right\} .
$$

$\tilde{N}$ is a closed symmetric $T$-invariant subset of $X \times X$ and it is easy to see that $(X, T)$ is uniformly rigid with respect to $\left\{n_{k}\right\}$ iff $N=\Delta$ (where $\Delta=\{(x, x): x \in X\}$ ). The analogous regionally proximal relation $Q$ which is defined for an arbitrary minimal flow $(X, T)$ by

$$
Q=\left\{\begin{array}{cc}
\text { There exists a sequence }\left\{m_{j}\right\} \text { and } \\
\left(x, x^{\prime}\right): & \begin{array}{c}
\text { sequences } x_{j} \rightarrow x \text { and } x_{j}^{\prime} \rightarrow x^{\prime} \text { with } \\
d\left(T^{m_{j}} x_{j}, T^{m_{j}} x_{j}^{\prime}\right) \rightarrow 0
\end{array}
\end{array}\right\}
$$

is of fundamental importance in the abstract theory of topological dynamics. Surprisingly $Q$ turns out to be an equivalence relation. Thus for a minimal flow $(X, T)$ to be equicontinuous it is necessary and sufficient that $Q=\Delta$ and the quotient flow $(X / Q, T)$ is the largest equicontinuous factor of $(X, T)$. A necessary and sufficient condition for $(X, T)$ to have only trivial equicontinuous factors can be derived, namely that it is (topologically) weakly mixing. In particular when $(X, T)$ is distal it always admits a non-trivial equicontinuous factor. (See [F, V, P, Ke-R, E-Ke, B] for these results). As we shall see minimal distal flows are weakly rigid and there are other analogies between distal and rigid flows. Thus we are naturally led to the following two questions

(1) Is $\tilde{N}$ always an equivalence relation?

(2) Does a minimal rigid (weakly rigid) flow always have a non-trivial uniformly rigid factor?

In order to describe our results concerning the first question we need some more definitions. In a minimal flow $(X, T)$ let $P=\left\{\left(x, x^{\prime}\right): \bar{O}\left(x, x^{\prime}\right) \supset \Delta\right\}$,

$$
L=\left\{\left(x, x^{\prime}\right): \bar{O}\left(x, x^{\prime}\right) \text { contains } \Delta \text { as a unique minimal subset }\right\}
$$

( $\bar{O}$ denotes orbit closure). It is easy to see that $P$ and $L$ are symmetric and $T$-invariant, $L \subset P$ and $L$ is an equivalence relation on $X,[C] . P$ and $L$ are called respectively the proximal and syndetically proximal relations on $(X, T) .(X, T)$ is distal iff $P=\Delta$ and we say that $(X, T)$ is syndetically distal if $L=\Delta$. Thus every distal flow is syndetically distal. In $\S 2$ we show that $\tilde{N} \subset L$. Hence a syndetically distal (and in particular a distal) rigid flow is uniformly rigid. (We wish to thank B. Weiss who is a co-author of this result for his permission to include it in our paper.) The next two sections are devoted to a general method of construction of minimal flows with various properties. In particular we retrieve Körner's example of a minimal rigid but not uniformly rigid flow in a simpler and more transparent way and construct an example in which $\tilde{N}$ is not an equivalence relation, answering question (1) above in the negative. We also investigate the structure of these examples, showing that they are almost one to one extensions of an equicontinuous flow - i.e. almost automorphic flows. In particular they admit non-trivial uniformly rigid factors. We have no complete answer to our second question. In the last section we collect some miscellaneous results about rigidity: Distal flows are weakly rigid, there are distal 
flows which are not rigid; (thus in general (i) $\Rightarrow$ (ii)). Rigid flows have zero topological entropy and there exist many uniformly rigid weakly mixing minimal flows. Finally mixing flows admit only trivial rigid factors.

\section{The relations $N$ and $\tilde{N}$ on a rigid flow}

Let $(X, T)$ be a rigid flow with respect to the sequence $\left\{n_{k}\right\}$. Put

$$
N=\left\{\left(x, x^{\prime}\right): \begin{array}{c}
\text { There exist a sequence } x_{k} \rightarrow x \\
\text { and subsequence }\left\{n_{k}^{\prime}\right\} \text { of }\left\{n_{k}\right\} \\
\text { such that } T^{n_{k}^{\prime}} x_{k} \rightarrow x^{\prime}
\end{array}\right\} .
$$

Clearly $N$ is closed and $T$-invariant. The following proposition is easy to verify.

Proposition 2.1

(1) $N \subset \tilde{N} \subset N \circ N^{-1}$ (where $N^{-1}=\{(x, y):(y, x) \in N\}$ ).

(2) $(X, T)$ is uniformly rigid iff $\tilde{N}=\Delta$. (We note that it is possible to build an example where $\tilde{N} \neq N \circ N^{-1}$.)

Proposition 2.2. Let $(X, T)$ be a minimal rigid flow, then

(1) $\tilde{N} \subset L$; thus $\left(x, x^{\prime}\right) \in \tilde{N}$ implies $\bar{O}\left(x, x^{\prime}\right) \subset P$.

(2) There exists a dense $G_{\delta}$ subset $X_{0} \subset X$ with $X_{0} \times X \cap N=\Delta$ hence $X_{0} \times X_{0} \cap \tilde{N}=\Delta$. In particular $N$ and $\tilde{N}$ are meagre subsets of $X \times X$.

Proof. (1) Since $L$ is an equivalence relation it suffices to show that $N \subset L$, for then by Proposition $2.1 \tilde{N} \subset N \circ N^{-1} \subset L \circ L^{-1} \subset L$. Moreover since $N$ is closed and $T$-invariant $N \subset L$ will follow from $N \subset P$.

Suppose then that $\left(x, x^{\prime}\right) \in N \backslash P$ and let $\delta=\inf \left\{d\left(T^{n} x, T^{n} x^{\prime}\right): n \in \mathbb{Z}\right\}>0$. Since $\left(x, x^{\prime}\right) \in N$ there exist a sequence $x_{k} \rightarrow x$ and a subsequence $\left\{n_{k}^{\prime}\right\}$ of $\left\{n_{k}\right\}$ with $T^{n_{k}^{\prime}} x_{k} \rightarrow x^{\prime}$. Let $U \subset X$ be a non-empty open set. By minimality there exists $l$ with $T^{l} x \in U$. Since $T_{k}^{n_{k}} T^{l} x_{k} \rightarrow T^{l} x^{\prime}$ and $T^{l} x_{k} \rightarrow T^{l} x$ we have for $k$ large enough $T^{l} x_{k} \in U$ and $\delta<d\left(T^{n_{k}^{\prime}} T^{l} x_{k}, T^{l} x_{k}\right)$.

Thus the open set $V_{k_{0}}=\left\{z: \exists k>k_{0} d\left(T^{n_{k}} z, z\right)>\delta\right\}$ is dense and $B=\bigcap_{k=1}^{\infty} V_{k}$ is a dense $G_{\delta}$ subset of $X$. However for $z \in B, T^{n_{k} z} \rightarrow z$ a contradiction. Thus $N \subset P$ and the proof of (1) is complete.

(2) Put $V_{\varepsilon, k}=\left\{x: d\left(T^{n_{j}} x, x\right) \leq \varepsilon\right.$ for every $\left.j \geq k\right\}$. Then $\bigcup_{k} V_{\varepsilon, k}=X$ and by Bair's theorem the set $B_{\varepsilon}=\bigcup_{k=1}^{\infty}$ int $\left(V_{\varepsilon, k}\right)$ is an open dense subset of $X$.

We let $X_{0}=\bigcap_{k=1}^{\infty} B_{1 / k}$. Suppose $x \in X_{0}$ and $\left(x, x^{\prime}\right) \in N$, then there exist a subsequence $n_{k}^{\prime}$ of $n_{k}$ and a sequence $x_{k} \rightarrow x$ such that $T^{n_{k}^{\prime}} x_{k} \rightarrow x^{\prime}$. Assume $x \neq x^{\prime}$; choose $l$ with $1 / l<(1 / 2) d\left(x, x^{\prime}\right)$. Since $x \in X_{0}$ there exists $j$ with $x \in \operatorname{int}\left(V_{1 / l, j}\right)$. For big enough $k x_{k} \in V_{1 / l, j}$ and we have

$$
2 / l<d\left(x, x^{\prime}\right) \leq d\left(x, x_{k}\right)+d\left(x_{k}, T^{n_{k}^{\prime}} x_{k}\right)+d\left(T^{n_{k}^{\prime}} x_{k}, x^{\prime}\right) .
$$

Sending $k$ to infinity, we have the first and last terms on the right tend to zero while the middle term is less than $1 / l$. This contradiction shows that $\left(X_{0} \times X\right) \cap N=\Delta$. It follows that $N^{-1} \cap\left(X \times X_{0}\right)=\Delta$ and hence $N \circ N^{-1} \cap\left(X_{0} \times X_{0}\right)=\Delta$.

COROLlARY 2.3. For a minimal syndetically distal (and hence also distal) flow, rigidity is equivalent to uniform rigidity. 
Remark. Chacon's flow is one example of a minimal syndetically distal non-distal flow. However, since it is zero dimensional it cannot be rigid (see § 6.7).

\section{Concatenation flows}

Let $I$ denote the interval $[-1,1]$, and let $\Omega=I^{Z}$ be the compact metric space of bidirectional sequences in $I$, with the metric

$$
d(x, y)=\sup _{n \in Z} 2^{-|n|}\left|x_{n}-y_{n}\right| .
$$

We call elements of $I^{n} n$-strings and we let $\left\|w, w^{\prime}\right\|=\sup _{1 \leq i \leq n}\left|w_{i}-w_{i}^{\prime}\right|$ for $w$, $w^{\prime} \in I^{n}$. If $x \in \Omega$ (or $x \in I^{n}$ ) and $i, k$ are integers, $i \leq k$, we let $x[i, k]$ be the string $w=x_{i} x_{i+1} \cdots x_{k}$. When $w \in I^{n}$ and $w^{\prime} \in I^{\prime}$ then $w w^{\prime}$ is the $(n+l)$-string $w_{1} w_{2} \cdots w_{n} w_{1}^{\prime} \cdots w_{l}^{\prime}$. We say that an $n$-string $w$ appears in $x \in \Omega$ (or $x \in I^{l}$ ) at the $j$-the coordinate if $x[j, j+n-1]=w$. If $W \subset I^{n}, V \subset W^{2}$ are closed subsets and $i$, $l>2$ are integers, we define

$$
\begin{aligned}
C_{i}(W, V) & =\{x \in \Omega: \forall j \in \mathbb{Z}, x[i+j n, i+j n+2 n-1] \in V\}, \\
C(W, V) & =\bigcup_{i=0}^{n-1} C_{i}(W, V), \\
C(W, V, l) & =\left\{x \in W^{l}: \forall 0 \leq j<l-2, x[j n+1, j n+2] \in V\right\} .
\end{aligned}
$$

We will call $W$ the set of $n$-blocks and $V$ the set of legitimate pairs of $n$-blocks.

Definition 3.1 Let $\left\{n_{k}\right\}_{k=1}^{\infty}$ be a sequence of natural numbers, $W_{0}=I$ and for every $k$ let $W_{k} \subset C\left(W_{k-1}, V_{k-1}, n_{k}\right)$ be compact sets. Let $V_{k} \subset C\left(W_{k-1}, V_{k-1}, 2 n_{k}\right)$. The subshift $(X, T)$ where $X=\bigcap_{k=1}^{\infty} C\left(W_{k}, V_{k}\right)$ will be called the concatenation flow of $\left\{W_{k}\right\},\left\{V_{k}\right\}$. In other words, $x \in X$ iff for every natural $k$ there are $\left\{w_{i}\right\}_{i=-\infty}^{\infty} \in W_{k}$ s.t. $x=\cdots w_{-1} w_{0} w_{1} w_{2} \cdots$ and s.t. for every integer $i, w_{i} w_{i+1} \in V_{k}$.

Definition 3.2. Let $(X, T)$ be the concatenation flow of $\left\{W_{k}\right\},\left\{V_{k}\right\}$ and let $m_{k}$ denote the length of the strings in $W_{k}$. Given $x \in X$, by Definition 3.1 we can find integers $t_{k}, k=1,2, \ldots$, such that $x \in C_{t_{k}}\left(W_{k}, V_{k}\right)$. We will call $t_{k}$ a $W_{k}$ partition of $x$. One can easily see that we can choose $\left\{t_{k}\right\}_{k=1}^{\infty}$ such that $t_{k-1} \equiv t_{k}\left(\bmod m_{k-1}\right)$ for every $k$. Such a sequence $\left\{t_{k}\right\}$ will be called a block partition of $X$. If we choose $\left\{t_{k}\right\}$ s.t. $0 \leq t_{k} \leq m_{k}$ we will say that $\left\{t_{k}\right\}$ is a normalized block partition of $x$.

Definition 3.3. Let $t$ be a $W_{k}$ partition of $x \in X$ such that $-m_{k} / 2<t \leq m_{k} / 2$. $v=x\left[t-m_{k}, t+m_{k}-1\right] \in V_{k}$ will be called a central $V_{k}$ block of $X$.

The proof of the next lemma is left to the reader.

Lemma 3.4. Let $W_{k}, V_{k}, n_{k}, k=1,2, \ldots$, and $(X, T)$ be as in Definition 3.1. Suppose that the following condition holds: for every natural $k$ there exists a $2^{-k}$ net $\left\{u_{1}^{(k)}, u_{2}^{(k)}, \ldots, u_{l_{k}}^{(k)}\right\}$ of $V_{k-1}$ such that, for every $w \in W_{k}, u_{i}^{(k)}$ appears in $w$ of every $1 \leq i \leq l_{k}$. Then $(X, T)$ is a minimal flow.

Definition 3.5. Let $(X, T)$ be the concatenation flow of $\left\{W_{k}\right\},\left\{V_{k}\right\}, W_{k} \subset I^{m_{k}}$, satisfying the following four conditions:

(1) For each natural $k$ there exists $1<r_{k}<m_{k} / 2$ and $\eta_{k} \in I^{r_{k}}$ such that for every $w \in W_{k}, w\left[1, r_{k}\right]=\eta_{k}$. 
(2) For every $w \in W_{k}$, where $w=w_{1} w_{2} \cdots w_{n_{k}}, w_{i} \in W_{k-1}$, and for every $i$ s.t. $(i-1) m_{k-1}>r_{k},\left\|w_{i-1}, w_{i}\right\|<2^{-k}$.

(3) Let $w$ be as in (2), then $w_{1}=w_{2}=w_{n_{k-1}}=w_{n_{k}}$.

(4) For every natural $k$, a $2^{-k}$ net of $V_{k-1}$ appears in $\eta_{k}$.

We call such a flow a concatenation flow with fixed part $\left\{\eta_{k}\right\}$.

Proposition 3.6. Let $(X, T)$ be a concatenation flow with a fixed part $\left\{\eta_{k}\right\}$ then $(X, T)$ is a minimal rigid flow (with respect to the sequence $m_{k}$ ).

Proof. From Lemma 3.4, it is clear that $(X, T)$ is minimal. It is sufficient to show that for every $\varepsilon>0$, every integer $i$ and every $x \in X$ there exists $k_{0}$ such that for every $k>k_{0},\left|x[i]-T^{m_{k}} x[i]\right|<\varepsilon$. Let $\left\{t_{k}\right\}$ be a block partition of $x$. Define $w_{k}^{(j)}=$ $x\left[t_{k}+j m_{k}, t_{k}+j m_{k}+m_{k}-1\right] \in W_{k}$ and suppose that $t_{k}+j_{k} m_{k} \leq i<t_{k}+j_{k} m_{k}+m_{k}$, or, in other words $x[i]$ is in $w_{k}^{\left(j_{k}\right)}$.

We consider two cases.

(1) There exists no $k$ such that $x[i]$ is contained in the fixed part $\eta_{k}$ of $w_{k}^{\left(j_{k}\right)}$. In this case, choose $k_{0}$ such that $2^{-k_{0}}<\varepsilon$. For every $k>k_{0}$ we have by condition (2) of Definition 3.5 (or by condition (3), if $w_{k}^{\left(j_{k}\right)}$ is the last $m_{k}$ string of $w_{k+1}^{\left(j_{k}{ }^{\prime}\right)}$ ) that $\left\|w_{k}^{\left(j_{k}\right)}, w_{k}^{\left(j_{k}+1\right)}\right\|<2^{-k}<\varepsilon$ and thus, $\left|x[i]-x\left[i+m_{k}\right]\right|<\varepsilon$.

(2) There exists $k_{0}$ such that $x[i]$ is contained in the fixed part $\eta_{k_{0}}$ of $w_{k_{0}}^{\left(j_{k_{0}}\right)}$. In this case, the fact that $m_{k_{0}}$ divides $m_{k}$ for every $k>k_{0}$, implies that shifting $x$ [i] by $m_{k}$ indices brings us to the same place in $\eta_{k_{0}}$ (in another $m_{k_{0}}$ block). Thus, $x[i]=x\left[i+m_{k}\right]$.

So in each case, we have found the required $k_{0}$.

LemMa 3.7. Let $(X, T)$ be a concatenation flow with a fixed part $\eta_{k}$ and let $x, x^{\prime} \in X$ have the same normalized block partition $\left\{t_{k}\right\}$, where $t_{k} \rightarrow \infty$ and $t_{k}-m_{k} \rightarrow-\infty$. Let $w_{k}=x\left[t_{k}-m_{k}, t_{k}-1\right]$ and $w_{k}^{\prime}=x^{\prime}\left[t_{k}-m_{k}, t_{k}-1\right]$. If for every natural $k, w_{k} w_{k}^{\prime}$ appears in $\eta_{k+1}$ then $\left(x, x^{\prime}\right) \in N$.

Proof. Choose $y \in X$. For every natural $k, \eta_{k}$ appears in $y$. Thus, we can find for each $k$ an integer $s_{k}$ such that for $x_{k}=T^{s_{k}} y$ we have

$$
x_{k}\left[t_{k}-m_{k}, t_{k}+m_{k}-1\right]=w_{k} w_{k}^{\prime} \text {. }
$$

Clearly, $x_{k} \rightarrow x$ and $T^{m_{k}} x_{k} \rightarrow x^{\prime}$ and thus $(x, x) \in N$.

Note that we have almost no restriction when choosing the fixed part $\eta_{k}$ and thus we can arrange that any given $w_{k} w_{k}^{\prime}$ will appear in $\eta_{k}$.

4. The structure of concatenation flows with fixed part

In this section we show that certain concatenation flows with fixed part are almost automorphic.

LEMMA 4.1. Let $(X, T)$ be a concatenation flow with a fixed part $\left\{\eta_{k}\right\}$. Suppose that, for a natural number $k$, a string $w_{k} w_{k}^{\prime}$ appears in $\eta_{k}$ such that $w_{k}, w_{k}^{\prime} \in W_{k-1}$ and $\left\|w_{k}, w_{k}^{\prime}\right\|>2^{-k}$. Under these conditions every $x \in X$ has only one normalized $W_{k}$ partition.

Proof. Let $x \in X$ and $k$ such that in $\eta_{k}$ appears the string $w_{k} w_{k}^{\prime}$, where $\left\|w_{k}, w_{k}^{\prime}\right\|>2^{-k}$ 
and $w_{k}, w_{k}^{\prime} \in W_{k-1}$. Let $r_{k}$ be the length of the string $\eta_{k}$ and let $1 \leq i_{k}<r_{k}$ be the minimal index such that $\left|\eta_{k}\left[i_{k}\right]-\eta_{k}\left[i_{k}+m_{k}-1\right]\right|>2^{-k}$.

Suppose that $x$ has two normalized $W_{k}$ partitions $t$ and $t^{\prime}$, and $t<t^{\prime}$. We consider the two following cases:

(1) $t^{\prime}-t<r_{k}$. We have $x\left[t, t+r_{k}-1\right]=\eta_{k}=x\left[t^{\prime}, t^{\prime}+r_{k}-1\right]$ and so $\mid x\left[t+i_{k}\right]-$ $x\left[t+i_{k}+m_{k-1}\right] \mid>2^{-k}$. Since outside the fixed part $\eta_{k},\left|x[i]-x\left[i+m_{k-1}\right]\right|<2^{-k}$, $t^{\prime}$ must be such that $x\left[t+i_{k}\right]$ lies in the fixed part $\eta_{k}$ of a $W_{k}$ block in the $t^{\prime}$ partition. This implies that $t^{\prime} \leq t+i_{k}<t^{\prime}+r_{k}$. (As $t^{\prime}-t<r_{k}$ we cannot have $t^{\prime}-m_{k} \leqq t+i_{k}<t^{\prime}-m_{k}+r_{k}$.) Suppose $t+i_{k}=t^{\prime}+j_{k}$. Then $j_{k}<i_{k}$ because $t<t^{\prime}$. But

$$
\left|\eta_{k}\left[j_{k}\right]-\eta_{k}\left[j_{k}+m_{k-1}\right]\right|=\left|x\left[t+i_{k}\right]-x\left[t+i_{k}+m_{k-1}\right]\right|>2^{-k}
$$

and this contradicts the minimality of $i_{k}$.

(2) $t^{\prime}-t \geq r_{k}$. We have $\left|x\left[t^{\prime}+i_{k}\right]-x\left[t^{\prime}+i_{k}+m_{k-1}\right]\right|>2^{-k}$. From this it follows that also in the $t$ partition $x\left[t^{\prime}+i_{k}\right]$ must be in $\eta_{k}$ and thus $t+m_{k} \leq t^{\prime}+i_{k}<t+m_{k}+r_{k}$. (As $t^{\prime}-t \geq r_{k}$, we cannot have $t \leq t^{\prime}+i_{k}<t+r_{k}$.) Suppose $t_{k}^{\prime}+i_{k}=t_{k}+m_{k}+j_{k}$ then $j_{k}<i_{k}$ and again we have a contradiction.

Definition 4.2. Let $(X, T)$ be a minimal concatenation flow of $\left\{W_{k}\right\},\left\{V_{k}\right\}$ where $W_{k} \in I^{m_{k}}$ and where each $x \in X$ has only one normalized block partition. $\hat{X}$ will be the set of all sequences $\left\{t_{k}\right\}$ where $\left\{t_{k}\right\}$ is a normalized block partition of some $x \in X$. On $\hat{X}$ we will define the following metric: for $\left\{t_{k}\right\},\left\{t_{k}^{\prime}\right\} \in \hat{X}, d\left(t_{k}, t_{k}^{\prime}\right)=2^{-n}$ where $n=\min _{j=1,2 \ldots}\left\{j \mid t_{j} \neq t_{j}^{\prime}\right\}$. We will define $T: \hat{X} \rightarrow \hat{X}$ as follows: for $\left\{t_{k}\right\} \in \hat{X}, T\left\{t_{k}\right\}=\left\{t_{k}^{\prime}\right\}$ where $t_{k}^{\prime}=t_{k}+1\left(\bmod m_{k}\right)$. Clearly, $(\hat{X}, T)$ is an equicontinuous flow since $d\left(T\left\{t_{k}\right\}, T\left\{t_{k}^{\prime}\right\}\right)=d\left(\left\{t_{k}\right\},\left\{t_{k}^{\prime}\right\}\right)$.

Proposition 4.3. Let $(X, T)$ be a concatenation flow with a fixed part $\eta_{k}$. Suppose that there exists a natural number $k_{0}$ such that for every $k \geq k_{0}$ there exists $w_{k}, w_{k}^{\prime} \in W_{k-1}$ such that $\left\|w_{k}, w_{k}^{\prime}\right\|>2^{-k}$ and such that the string $w_{k} w_{k}^{\prime}$ appears in $\eta_{k}$. Then

(1) $(\hat{X}, T)$ is the maximal equicontinuous factor of $(X, T)$.

(2) $P=Q=\left\{\left(x, x^{\prime}\right) \in X \times X \mid x\right.$ and $x^{\prime}$ have the same block partition $\}$.

(3) $(\hat{X}, T)$ is an almost 1-1 factor of $(X, T)$.

Proof. By Lemma 4.1 for every $k>k_{0}$ and every $x \in X$ there is a unique normalized $W_{k}$ partition $t_{k}$ of $x$. Let $\left\{t_{k}^{\prime}\right\}_{k=1}^{\infty}$ be a normalized block partition of $x$. For $k \geq k_{0}$, $t_{k}^{\prime}=t_{k}$ and for $k<k_{0}, t_{k}^{\prime} \equiv t_{k_{0}}^{\prime}\left(\bmod m_{k}\right)$. So $\left\{t_{k}^{\prime}\right\}$ is unique and $(\hat{X}, T)$ is well defined.

Define $p: X \rightarrow \hat{X}, p(x)=\left\{t_{k}\right\}$, where $\left\{t_{k}\right\}$ is the normalized block partition of $x$. Clearly $p(T x)=T(p x)$. We will now prove that $p$ is continuous. Let $x \in X,\left\{x_{n}\right\} \in X$, $x_{n} \rightarrow x$. From Lemma 4.1, we have that $\left\{C_{i}\left(W_{k}, V_{k}\right)\right\}_{i=0}^{m_{k}-1}$ are pairwise disjoint sets for $k \geq k_{0}$.

Let $\varepsilon=\min _{0 \leq i_{1}<i_{2}<m_{k}} d\left(C_{i_{1}}\left(W_{k}, V_{k}\right), C_{i_{2}}\left(W_{k}, V_{k}\right)\right.$. Choose $n_{0}$ such that for every $n>n_{0}, d\left(x_{n}, x\right)<\varepsilon$. Suppose that $x \in C_{i_{0}}\left(W_{k}, V_{k}\right)$, then, for $n>n_{0}, x_{n} \in C_{i_{0}}\left(W_{k}, V_{k}\right)$. But this implies that, for $n>n_{0}, d\left(p\left(x_{n}\right), p(x)\right)<2^{-k}$ and so $p$ is continuous.

We will now prove that $(\hat{X}, T)$ is an almost one to one factor of $(X, T)$.

Let $\left\{y_{k}\right\}=y \in \hat{X}$ be such that $y_{k}=0$ for each natural $k$, and let $x \in X$ be such that $x \in p^{-1}(y)$. For every $k, x\left[0, m_{k}-1\right] \in W_{k}$ and thus $x\left[0, r_{k}-1\right]=\eta_{k}$. By (3) in Definition 3.5

$$
x\left[-m_{k-1},-1\right]=x\left[0, m_{k-1}-1\right]=\eta_{k}\left[1, m_{k-1}\right]
$$


But this determines $x$. Thus $p^{-1}(y)$ contains only one point and $(\hat{X}, T)$ is an almost one to one factor of $(X, T)$.

It also follows that $(\hat{X}, T)$ is the maximal equicontinuous factor of $(X, T)$, because an equicontinuous flow has no proper almost one to one factors. Clearly now $P \subset Q \subset R$ where $R=\left\{\left(x, x^{\prime}\right) \mid p(x)=p\left(x^{\prime}\right)\right\}$. But if $\left(x, x^{\prime}\right) \in R$, they are proximal because, for each natural $k, \eta_{k}$ appears at the same places in $x$ and $x^{\prime}$, so $P=Q=R$.

CoRollary 4.4. If $N \neq \Delta$ in a concatenation flow $X$ with fixed part, then every $x \in X$ has a unique normalized block partition. Moreover, $\left(x, x^{\prime}\right) \in N$ implies that $x$ and $x^{\prime}$ have the same block partition.

Proof. Suppose $x_{k} \rightarrow x$ and $T^{m_{k}} x_{k} \rightarrow x^{\prime}$. Choose $k$ big enough such that $\left|x[i]-x^{\prime}[i]\right|>$ $2^{-k+2}$ for some $i$. Let $n_{0}, n_{0}>k$, be such that for $n>n_{0}\left|x_{n}[i]-x[i]\right|<2^{-k}$ and $\left|x_{n}\left[i+m_{n}\right]-x^{\prime}[i]\right|<2^{-k}$. Then $\left|x_{n}[i]-x_{n}\left[i+m_{n}\right]\right|>2^{-k}>2^{-n}$ and $x_{n}[i]$ and $x_{n}\left[i+m_{n}\right]$ must lie in $\eta_{n+1}$. Hence the conditions of Proposition 4.3 are fulfilled (where $w_{k}$ is the $W_{n}$ block in $\eta_{n+1}$ that contains $x[i]$, and $w_{k}^{\prime}$ is the $W_{n}$ block that contains $\left.x\left[i+m_{n}\right]\right)$.

\section{Examples}

We will now build concrete examples of minimal rigid but not uniformly rigid concatenation flows with fixed part. The first example is an example where $N$ is not an equivalence relation and where for every $x \in X$ there exists at most one $x^{\prime} \neq x \in X$ such that $\left(x, x^{\prime}\right) \in N$.

Example 5.1. Let $f: I \rightarrow I$ be a monotonic ascending and continuous function. Choose $q, q^{\prime} \in I, q>q^{\prime}$. We will define by induction numbers $m_{k}$ and continuous monotonic functions $w_{k}: I \rightarrow I^{m_{k}}$ as follows: $w_{0}(s)=f(s) \forall s \in I$. Assume that we defined $w_{k-1}(s): I \rightarrow I^{m_{k-1}}$. Choose $\varepsilon>0$ s.t. $\left|s-s^{\prime}\right|<\varepsilon$ implies $\left\|w_{k-1}(s), w_{k-1}\left(s^{\prime}\right)\right\|<2^{-k}$ and choose $0=s_{1}<s_{2}<\cdots<s_{l_{k}}=1$ s.t. $\left|s_{i+1}-s_{i}\right|<\varepsilon, 1 \leq i \leq l_{k}$ and s.t. there exist $l_{k} \geq r>r^{\prime} \geq 1$ with $s_{r}=q, s_{r^{\prime}}=q^{\prime}$. Define

$$
\begin{aligned}
\eta_{k}= & w_{k-1}(0) w_{k-1}\left(s_{1}\right) w_{k-1}\left(s_{2}\right) \cdots w_{k-1}\left(s_{r}\right) w_{k-1}\left(s_{r^{\prime}}\right) w_{k-1}\left(s_{r^{\prime}+1}\right) \cdots \\
& w_{k-1}\left(s_{l_{k}}\right) w_{k-1}\left(s_{l_{k}-1}\right) \cdots w_{k-1}(0) w_{k-1}(0) .
\end{aligned}
$$

Define $\quad w_{k}(s)=\eta_{k} w_{k-1}\left(s s_{1}\right) w_{k-1}\left(s s_{2}\right) \cdots w_{k-1}\left(s s_{l_{k}}\right) w_{k-1}\left(s s_{l_{k-1}}\right) \cdots w_{k-1}(0) w_{k-1}(0)$ and let $m_{k}$ be the length of $w_{k}(s)$.

Define $W_{k}=\left\{w_{k}(s) \mid s \in I\right\}$ and

$$
V_{k}=\left\{w_{k}\left(s s_{i}\right) w_{k}\left(s s_{j}\right) \mid s \in I, 1 \leq i<l_{k}, j=i+1, i-1\right\} \cup\left\{w_{k}(q) w_{k}\left(q^{\prime}\right)\right\} .
$$

Define $(X, T)$ as the concatenation flow defined by $\left\{W_{k}\right\},\left\{V_{k}\right\}$.

Proposition 5.2. $(X, T)$ in Example 5.1 is a non-empty concatenation flow with a fixed part and thus is minimal and rigid.

Proof. Clearly for each $k, C\left(W_{k}, V_{k}\right)$ is not empty and thus $X \neq \varnothing$. $\eta_{k}$ contains $w_{k-1}\left(s_{i}\right) w_{k-1}\left(s_{i+1}\right)$ for every $1 \leq i<l_{k}$ and contains $w_{k-1}(q) w_{k-1}\left(q^{\prime}\right)$. By the definition of $\left\{s_{i}\right\}, \eta_{k}$ contains a $2^{-k}$ net of $V_{k-1} . \eta_{k}$ is a fixed part of $W_{k}$. By Proposition 3.6 $(X, T)$ is minimal and rigid.

LEMMA 5.3. $(X, T)$ is not uniformly rigid with respect to $m_{k}$. 
Proof. Let $x, x^{\prime} \in X$ be as follows.

We define by induction on $k x\left[i_{k}, j_{k}\right], x^{\prime}\left[i_{k}, j_{k}\right]$, where $i_{k}<i_{k-1}$ and $j_{k}>j_{k-1}$. For $k=0$, let $i_{0}=j_{0}=0$, and let $x\left[i_{0}, j_{0}\right]=w_{0}(q), x^{\prime}\left[i_{0}, j_{0}\right]=w_{0}\left(q^{\prime}\right)$. Assume that $x\left[i_{k}, j_{k}\right]\left(x^{\prime}\left[i_{k}, j_{k}\right]\right)$ is defined and equals $w_{k}(q)\left(w_{k}\left(q^{\prime}\right)\right)$. Consider $w_{k+1}(q)=$ $\eta_{k+1} w_{k}\left(s_{1} q\right) \cdots w_{k}\left(s_{l_{k}} q\right) \cdots w_{k}(0)$. As $s_{l_{k}}=1, w_{k}(q)$ appears in $w_{k+1}(q)$ (outside of $\left.\eta_{k+1}\right)$.

Define $i_{k+1}, j_{k+1}$ and $x\left[i_{k+1}, j_{k+1}\right]$ such that $x\left[i_{k+1}, j_{k+1}\right]=w_{k+1}(q)$ and such that $x\left[i_{k}, j_{k}\right]$ is the above mentioned appearance of $w_{k}(q)$ in $w_{k+1}(q)$. Define $x^{\prime}\left[i_{k+1}, j_{k+1}\right]$ to be $w_{k+1}\left(q^{\prime}\right)$. Notice that $x^{\prime}\left[i_{k+1}, j_{k+1}\right]$ is well defined, since $w_{k}\left(q^{\prime}\right)\left(=x^{\prime}\left[i_{k}, j_{k}\right]\right)$ appears in $w_{k+1}\left(q^{\prime}\right)$ exactly in the same place as $w_{k}(q)$ appears in $w_{k+1}(q)$.

Clearly, $i_{k+1}<i_{k}$ and $j_{k+1}>j_{k}$, which implies that all coordinates of $x, x^{\prime}$ are defined. Also $x, x^{\prime}$ have the same block partition. The construction implies that $x \neq x^{\prime}$ since $x[0]=w_{0}(q)=f(q) \neq f\left(q^{\prime}\right)=w_{0}\left(q^{\prime}\right)=x^{\prime}[0]$. For each $k, w_{k}(q)$ is a central $W_{k}$ block of $x$, and $w_{k}\left(q^{\prime}\right)$ is a central $W_{k}$ block of $x^{\prime} . w_{k}(q) w_{k}\left(q^{\prime}\right)$ appears in $\eta_{k}$ and thus by Lemma 3.7. $\left(x, x^{\prime}\right) \in N$ and by Proposition $2.1(X, T)$ is not uniformly rigid.

Proposition 5.4. Let $X$ be as in Example 5.1 then

(1) For each $x \in X$ there is at most one $x^{\prime} \neq x$ such that $\left(x, x^{\prime}\right) \in N$.

(2) If $x \neq x^{\prime}$ and $\left(x, x^{\prime}\right) \in N$ then for every integer $i x[i] \geq x^{\prime}[i]$. Thus $\left(x^{\prime}, x\right) \notin N$ and $N$ is not an equivalence relation.

Proof. Let $\left(x, x^{\prime}\right) \in N$ and $x \neq x^{\prime}$. Let $m_{j_{k}}$ be a subsequence of $m_{k}$ and $\left\{x_{k}\right\} \in X, x_{k} \rightarrow x$

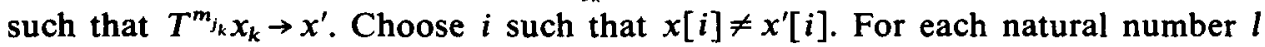
choose $i_{l}$ such that $x \in C_{i_{l}}\left(W_{l}, V_{l}\right)$ and such that $i_{l} \leq i \leq i_{l}+m_{l}$ i.e., $x\left[i_{l}, i_{l}+m_{l}-1\right]$ is the $W_{l}$ block $x$ in which $x[i]$ lies.

By 4.4. also $x^{\prime} \in C_{i_{1}}\left[W_{l}, V_{l}\right]$ and for $n$ big enough $x_{n} \in C_{i_{l}}\left(W_{l}, V_{l}\right)$. Denote $v_{l}=x\left[i_{l}, i_{l}+m_{l}-1\right], \quad v_{l}^{\prime}=x^{\prime}\left[i_{l}, i_{l}+m_{l}-1\right]$, and denote $v_{l}^{n}=x_{n}\left[i_{l}, i_{l}+m_{l}-1\right] \in W_{l}$, $v_{l}^{\prime n}=\left(T^{m_{j_{n}} x_{n}}\right)\left[i_{l}, i_{l}+m_{l}-1\right] \in W_{l}$. Choose $k$ s.t. $2^{-k}<\left|x[i]-x^{\prime}[i]\right| . x[i]$ is contained in $v_{k}$ and $v_{k}^{n} \rightarrow v_{k} . x^{\prime}[i]$ is contained in $v_{k}^{\prime}$ and $v_{k}^{\prime n} \rightarrow v_{k}^{\prime}$. As $\left|x[i]-x^{\prime}[i]\right|>2^{-k}$; we have, for large enough $n,\left\|v_{k}^{n}, v_{k}^{\prime n}\right\|>2^{-k}>2^{-j_{n}}$. For $j_{n}>k, v_{k}^{n}$ appears in $v_{j_{n}}^{n}$ and $v_{k}^{\prime n}$ appears in $v_{j_{n}}^{\prime n}$. Thus for $n$ big enough we have $\left\|v_{j_{n}}^{n}, v_{j_{n}}^{\prime n}\right\|>2^{-j_{n}} . v_{j_{n}}^{n}$ and $v_{j_{n}}^{\prime n}$ are two adjacent $W_{j_{n}}$ blocks in $x_{n}$. Thus the only possibility of their distance to be greater than $2^{-j_{n}}$ is that $v_{j_{n}}^{n}$ lies in $\eta_{j_{n}+1}$ and that $v_{j_{n}}^{n}=w_{j_{n}}(q), v_{j_{n}}^{\prime n}=w_{j_{n}}\left(q^{\prime}\right)$. We have $v_{k}^{n}$ appearing in $v_{k+1}^{n}$ appearing in $\cdots$ appearing in $v_{j_{n}-1}^{n}$ appearing in $v_{j_{n}}^{n}$. Thus for $k \leq l<j_{n}, v_{l}^{n} \neq v_{l}^{\prime n}$ which implies that $v_{l}^{n}$ does not lie in $\eta_{l+1}$. (Otherwise $v_{l}^{\prime n}$ would have also been in $\eta_{l+1}$ in the same place which implies $\left.v_{l}^{n}=v_{l}^{\prime n}\right)$. If we look at the definition of $w_{l}(q)$, this implies that there exists some number $s_{n}$ such that $v_{k}^{n}=$ $w_{k}\left(s_{n} q\right)$. Since $v_{k}^{\prime n}$ lies in $v_{j_{n}}^{\prime n}$ in the same place as $v_{k}^{n}$ lies in $v_{j_{n}}^{k}$, we have $v_{k}^{\prime n}=w_{k}\left(s_{n} q^{\prime}\right)$. Let $s=\varlimsup \varlimsup_{n}$ then $w_{k}(s q)=\lim _{n \rightarrow \infty} v_{k}^{n}=v_{k}$ and $v_{k}^{\prime}=\lim _{n \rightarrow \infty} v_{k}^{\prime n}=w_{k}\left(s q^{\prime}\right)$. Thus, we have

(*) $x\left[i_{k}, i_{k}+m_{k}-1\right]=w_{k}(s q)$

$\left({ }^{* *}\right) x^{\prime}\left[i_{k}, i_{k}+m_{k}-1\right]=w_{k}\left(s q^{\prime}\right)$.

As each coordinate in $w_{k}(s q)$ is greater than the corresponding one in $w_{k}\left(s q^{\prime}\right)$ we have $x[i]>x^{\prime}[i]$ which proves (2). 
Let $r_{k}$ denote the length of $\eta_{k}$ then $i>i_{k}+r_{k}$ (because $x\left[i_{k}, i_{k}+r_{k}-1\right]=$ $x^{\prime}\left[i_{k}, i_{k}+r_{k}-1\right]=\eta_{k}$ and $\left.x[i] \neq x^{\prime}[i]\right)$ and also $i_{k}+m_{k}-m_{k-1}>i$ (because

$$
\left.x\left[i_{k}+m_{k}-m_{k-1}, i_{k}+m_{k}-1\right]=x^{\prime}\left[i_{k}+m_{k}-m_{k-1}, i_{k}+m_{k}-1\right]=w_{k-1}(0)\right) .
$$

Thus $i_{k} \rightarrow-\infty$ and $i_{k}+m_{k} \rightarrow \infty$ and (**) defines $x^{\prime}$ uniquely.

In the first example, we had $N$ small with respect to $Q$. Now, we will show an example where $N=Q$.

Example 5.5. Define $(X, T)$ as in Example 5.1 changing $\eta_{k}$ to be as follows

$$
\eta_{k}=w_{k-1}(0) w_{k-1}(0) \eta_{k}^{\prime} w_{k-1}(0) w_{k-1}(0),
$$

where $\eta_{k}^{\prime}$ is the concatenation of $\left\{w_{k-1}\left(s_{j}\right) w_{k-1}\left(s_{i}\right)\right\}_{1 \leq i, j \leq l_{k}}$, and adding to $V_{k}$ the set $\left\{w_{k}\left(s_{i}\right) w_{k}\left(s_{j}\right)\right\}_{1 \leq i, j \leq l_{k}}$.

Proposition 5.6. Let $(X, T)$ be as in Example 5.5 and let $x$ and $x^{\prime}$ have the same block partition then $\left(x, x^{\prime}\right) \in N$.

Proof. Let $x, x^{\prime} \in X, x \neq x^{\prime}$ have the same normalized block partition $\left\{t_{k}\right\}$. The sequence $\left\{t_{k}\right\}$ is not bounded, because otherwise, as $x\left[t_{k}, t_{k}+r_{k}-1\right]=\eta_{k}=$ $x^{\prime}\left[t_{k}, t_{k}+r_{k}-1\right]$ and $x\left[t_{k}-m_{k-1}, t_{k}-1\right]=w_{k-1}(0)=x^{\prime}\left[t_{k}-m_{k-1}, t_{k}-1\right]$, we would have $x=x^{\prime}$ - a contradiction. Thus we may assume that $\left\{t_{k}\right\}$ is not bounded from below. (Otherwise, as $t_{k}<m_{k} / 2$ we have $t_{k}-m_{k}$ is not bounded from below. Replacing $t_{k}$ by $t_{k}-m_{k}$ we have $t_{k}$ bounded). As $t_{k}<m_{k} / 2, t_{k}+m_{k}$ is not bounded from above. Fix $k$. There exists $r$ such that for $s_{r},\left\|x\left[t_{k}, t_{k}+m_{k}-1\right], w_{k}\left(s_{r}\right)\right\|<2^{-k}$ and $r^{\prime}$ such that for $s_{r^{\prime}},\left\|x^{\prime}\left[t_{k}, t_{k}+m_{k}-1\right], w_{k}\left(s_{r^{\prime}}\right)\right\|<2^{-k}$.

Let $y \in X . w_{k}\left(s_{r}\right) w_{k}\left(s_{r^{\prime}}\right)$ appears in $\eta_{k+1}$ thus there exists $n$ such that $T^{n}(y)\left[t_{k}, t_{k}+m_{k}-1\right]=w_{k}\left(s_{r}\right)$ and $T^{n+m_{k}} y\left[t_{k}, t_{k}+m_{k}-1\right]=w_{k}\left(s_{r^{\prime}}\right)$. Define $x_{k}=T^{n} y$. As $t_{k}$ is not bounded from below and $t_{k}+m_{k}$ is not bounded from above, we have $x_{k} \rightarrow x$ and $T^{m_{k}} x_{k} \rightarrow x^{\prime}$.

Example 5.7. We will use the notations of Example 5.1. Let $q^{\prime \prime}, q, q^{\prime} \in I q^{\prime \prime}>q>q^{\prime}$. Let $s_{1}, s_{2} \cdots s_{l_{k}}$ be as in Example 5.1 and such that there exist $r^{\prime \prime}>r>r^{\prime}$ with $s_{r^{\prime \prime}}=q^{\prime \prime}$, $s_{r}=q, s_{r^{\prime}}=q^{\prime}$. Define

$$
\begin{aligned}
\eta_{k+1}= & w_{k}(0) w_{k}\left(s_{1}\right) w_{k}\left(s_{2}\right) \cdots w_{k}\left(s_{r}\right) w_{k}\left(s_{r^{\prime}}\right) w_{k}\left(s_{r^{\prime}+1}\right) \\
& \cdots w_{k}\left(s_{r^{\prime \prime}-1}\right) w_{k}\left(s_{r^{\prime \prime}}\right) w_{k}\left(s_{r^{\prime \prime}}\right) w_{k}\left(s_{r^{\prime \prime}-1}\right) \cdots \\
& w_{k}\left(s_{r^{\prime}}\right) w_{k}\left(s_{r^{\prime \prime}}\right) w_{k}\left(s_{r^{\prime \prime}+1}\right) \cdots w_{k}\left(s_{l_{k}}\right) w_{k}\left(s_{l_{k-1}}\right) \cdots w_{k}\left(s_{1}\right)
\end{aligned}
$$

and define

$$
\begin{gathered}
w_{k+1}(t)=\eta_{k+1} w_{k}\left(s_{1} t\right) \cdots w_{k}(0), \\
W_{k+1}=\left\{w_{k+1}(t) \mid t \in[0,1]\right\}, \\
V_{k+1}=\left\{w_{k}\left(t t_{i}\right) w_{k}\left(t t_{i+1}\right), t \in[0,1]\right\}_{i=1, l_{k}} \cup\left\{w_{k}\left(t_{i+1} t\right) w_{k}\left(t_{i} t\right), t \in[0,1]\right\}_{i=1, l_{k}} \\
\cup\left\{w_{k}(q) w_{k}\left(q^{\prime}\right)\right\} \cup\left\{w_{k}\left(q^{\prime}\right) w_{k}\left(q^{\prime \prime}\right)\right\} .
\end{gathered}
$$

Proposition 5.8. Let $(X, T)$ be as in Example 5.7 then the relation $\tilde{N}$ (with respect to $m_{k}$ ) is not an equivalence relation.

Proof. We will only sketch the proof. We will find $x, x^{\prime}, x^{\prime \prime}$ such that $\left(x, x^{\prime}\right) \in \tilde{N}$, 
$\left(x^{\prime}, x^{\prime \prime}\right) \in \tilde{N}$. But $\left(x, x^{\prime \prime}\right) \notin \tilde{N}$. Let $x, x^{\prime}, x^{\prime \prime}$ be such that for every $k$ their central $W_{k}$ block are $w_{k}(q), w_{k}\left(q^{\prime}\right), w_{k}\left(q^{\prime \prime}\right)$ respectively.

In a similar way to the proof of Proposition 5.4, one can show the following Lemmas:

(1) Let $x_{k}^{\prime \prime}$ be a sequence such that $x_{k} \rightarrow x^{\prime \prime}$ then $T^{m_{k}} x_{k}^{\prime \prime} \rightarrow x^{\prime \prime}$.

(2) Let $x_{k}$ be a sequence such that $x_{k} \rightarrow z$ and such that for a subsequence $m_{k}^{\prime}$ of $m_{k} T^{m_{k}^{\prime}} x_{k} \rightarrow x^{\prime \prime}$ then $z=x^{\prime}$ or $z=x^{\prime \prime}$.

From (1) and (2) it follows that $\left(x, x^{\prime \prime}\right) \notin \tilde{N}$. But clearly from Lemma 3.7, we have $\left(x, x^{\prime}\right) \in N \subset \tilde{N}$ and $\left(x^{\prime}, x^{\prime \prime}\right) \in N \subset \tilde{N}$.

\section{Miscellaneous results}

LEMMA 6.1. $(X, T)$ is weakly rigid iff the identity map e is a limit point of the enveloping semigroup $E(X)$ of $(X, T)$.

Proof. Follows directly from the definition of weak rigidity.

COROLLARY 6.2. Every minimal distal flow is weakly rigid.

Proof. By $[E](X, T)$ is distal iff $E(X)$ is a group. If $e$ is isolated in $E(X)$ then $X$ is finite, hence rigid.

Consider the distal minimal flow on $T^{2}$ defined by $T(x, y)=(x+\alpha, y+x)$, where $T=[0,1], \alpha$ an irrational number in $T$ and addition is mod 1 . We claim that this flow is not rigid. In fact if $T^{n_{k}}$ tend pointwise to the identity for some sequence $\left\{n_{k}\right\}$, then since $T^{n}(x, y)=(x+n \alpha, y+n x+[n(n-1) / 2] \alpha)$ we have $n_{k} x \rightarrow 0$ for every $x \in T$, an obvious absurdity. Thus, in general, weak rigidity does not imply rigidity.

Proposition 6.3. The topological entropy of a rigid flow is zero.

Proof. Let $(X, T)$ be rigid with respect to the sequence $\left\{n_{k}\right\}$. Then $T^{n_{k}} x \rightarrow x$ for every $x \in X$ and by the dominated convergence theorem we have for every invariant probability measure $\eta$ on $X$ and every $f \in L_{2}(\eta) . \lim T^{n_{k}} f=f$ in $L_{2}(\eta)$. Thus the measure preserving system $(X, \eta, T)$ is rigid in the measure theoretical sense. It follows from $[F W]$ that it has zero entropy. Thus the variational principle implies that the topological entropy of $(X, T)$ is zero as well.

One consequence of Furstenberg's structure theorem for distal flows is that the topological entropy of such flow vanishes [Ke]. Since every minimal distal flow is weakly rigid, this suggests the possibility that weak rigidity is a sufficient condition for zero entropy. We do not know whether this is true.

Considering the second question posed in the introduction, we observe that since an equicontinuous flow is uniformly rigid, a counter example to this question should be looked for among the weakly mixing flows. Recall that $(X, T)$ is (strongly) mixing if for every two non-empty open subsets $U, V$ of $X$ the set $N(U, V)=$ $\left\{n \in \mathbb{Z}: T^{n} U \cap V \neq \varnothing\right\}$ has a finite complement.

Proposition 6.4. A strongly mixing minimal flow admits only trivial rigid factors.

Proof. Suppose $(X, T)$ is minimal and strongly mixing; then every factor of $(X, T)$ have these same properties. Thus in order to prove our proposition, it suffices to show that $(X, T)$ is trivial if in addition to the above it is also rigid. Assume therefore 
that $(X, T)$ is rigid with respect to $\left\{n_{k}\right\}$. Let $\left(x, x^{\prime}\right) \in X \times X$ and $U$ and $V$ neighbourhoods of $x$ and $x^{\prime}$ respectively. By strong mixing there is $k_{0}$ such that $T^{n_{k}} U \cap V \neq \varnothing$ for $k \geq k_{0}$. Thus it is possible to find a subsequence $\left\{n_{k}^{\prime}\right\}$ of $\left\{n_{k}\right\}$ and a sequence $x_{k} \rightarrow x$ for which $T^{n}{ }_{k}^{\prime} x_{k} \rightarrow x^{\prime}$. In other words $X \times X \subset N$. But by Proposition 2.2 $N \subset L \subset P$. Since for every $x \in X T x \neq x$ implies $(x, T x) \notin P$ we conclude that $X$ has only one point.

We next show the existence of plenty of minimal, uniformly rigid, weakly mixing flows. Let $(Z, \sigma)$ be a minimal flow rigid with respect to a sequence $\left\{n_{k}\right\}$. Let $Y$ be a compact metric space. Let $X=Z \times Y$ and let $\mathscr{H}(X)$ be the group of homeomorphisms of $X$. Put

$$
\mathscr{O}(\sigma)=\left\{G^{-1} \circ \sigma \circ G: G \in \mathscr{H}(X)\right\}
$$

(we identify $\sigma$ with $\sigma \times$ id), and let

$$
V_{k_{0}, \varepsilon}=\left\{T \in \bar{O}(\sigma): \exists k \geq k_{0} \quad d\left(T^{k}, \text { id }\right)<\varepsilon\right\} .
$$

Clearly $V_{k_{0}, \varepsilon}$ is an open dense subset of $\overline{\mathcal{O}}(\sigma)$, and $\mathscr{R}_{1}=\bigcap_{k} V_{k, 1 / k}$ is a residual subset of $\bar{O}(\sigma)$. For each $T \in \mathscr{R}_{1},(X, T)$ is uniformly rigid w.r.t. some subsequence of $\left\{n_{k}\right\}$. If we assume that the identity path component of $\mathscr{H}(Y)$ acts minimally on $Y$ we get from [GW, Th. 1] the existence of a residual subset $\mathscr{R}_{2} \subset \bar{O}(\sigma)$ such that each member of $\mathscr{R}_{2}$ acts minimally on $X$. Finally assuming further that $Z=T$ and $\sigma=R_{\alpha}$, an irrational rotation, we get from [GW, Th. 5] a residual subset $\mathscr{R}_{3}$ of $\overline{\mathcal{O}}(\sigma)$ every member of which acts weak mixingly on $X$. Taking in the latter case $\mathscr{R}=\mathscr{R}_{1} \cap \mathscr{R}_{2} \cap \mathscr{R}_{3}$ we have:

Proposition 6.5. Let $Z=T, \sigma=R_{\alpha}$ an irrational rotation. Let $Y$ be a non-trivial compact metric space, let $X=Z \times Y$ and let $\mathscr{H}_{0}(Y)$ be the identity path component of $\mathscr{H}(Y)$. If the action of $\mathscr{H}_{0}(Y)$ on $Y$ is minimal then there exists a residual subset $\mathscr{R}$ of $\overline{\mathcal{O}}(\sigma)$ such that for each $T \in \mathscr{R}$ the flow $(X, T)$ is uniformly rigid, minimal and weakly mixing.

We conclude by showing that weak rigidity implies equicontinuity in zero dimensional flows.

LEMMA 6.6. Let $Y \subset\{0,1\}^{\mathbf{Z}}=\Omega$ be a T-invariant closed subset of the two shift $(\Omega, T)$. If $(Y, T)$ is infinite then there exist points $y_{0}, y_{1}, z_{0}, z_{1} \in Y$ such that $y_{0}(0)=z_{0}(0)=0$, $y_{1}(0)=z_{1}(0)=1, y_{0}(i)=y_{1}(i)$ for $i<0$ and $z_{0}(i)=z(i)$ for $i>0$.

Proof. Let $k$ be the minimal length such that for every block $B$ of length $k$ whenever $B$ appears in $y \in Y$ say, $y[n, n+k-1]=B$, then always $y(n+k)=0$ (or always $y(n+k)=1)$.

If $k<\infty$ then each $y \in Y$ is periodic of period $\leq L$, where $L$ is the number of $k$-blocks appearing in $Y$. This implies that $Y$ is finite contradicting our assumption. Thus $k=\infty$; let $\left|B_{i}\right|=k_{i}$, a sequence $n_{i}$ and points $y_{1}^{(i)}, y_{0}^{(i)} \in Y$ be chosen with $y_{\varepsilon}^{(i)}\left[n_{i}, n_{i}+k_{i}-1\right]=B_{i}, \quad \varepsilon=0, \quad 1$ and $y_{0}^{(i)}\left(n_{i}+k_{i}\right)=0, \quad y_{1}^{(i)}\left(n_{i}+k_{i}\right)=1$. We can assume the existence of the limits $y_{0}=\lim T^{-\left(n_{i}+k_{i}\right)} y_{0}^{(i)}, y_{1}=\lim T^{-\left(n_{i}+k_{i}\right)} y_{1}^{(i)}$. Then $y_{0}(0)=0, y_{1}(0)=1$ and $y_{0}(i)=y_{1}(i)$ for $i<0$. The existence of $z_{0}$ and $z_{1}$ follows similarly. 
Proposition 6.7. Let $(Y, T)$ be a minimal weakly rigid flow.

(i) If $(Y, T)$ is a subshift then $Y$ is finite.

(ii) If $Y$ is zero dimensional then $(Y, T)$ is equicontinuous.

Proof. (i) Let $\left\{T^{n_{\alpha}}\right\}$ be a net converging to the identity pointwise on $Y$. We can assume that $n_{\alpha}>0 \forall \alpha$. If $Y$ is infinite, there exist $y_{0}, y_{1} \in Y$ as in Lemma 6.6. However $\lim T^{n_{\alpha}} y_{0}=\lim T^{n_{\alpha}} y_{1}$ contradicting our assumption that $\lim T^{n_{\alpha}}=\mathrm{id}$.

(ii) Let $V \subset Y$ be a clopen set then the map $\pi_{V}: Y \rightarrow \Omega, \pi_{V}(y)=\left\{1_{V}\left(T^{n} y\right)\right\}_{n \in Z}$ is a homomorphism of $(Y, T)$ into $(\Omega, T)$. Since the image $(\pi(Y), T)$ is a weakly rigid subshift, (i) implies it is finite. Since by assumption the maps $\pi_{V}, V$ clopen in $Y$, separate points in $Y$, we have $(Y, T)=\lim _{\leftarrow}\left(\pi_{V}(Y), T\right)$. This implies the equicontinuity of $(Y, T)$.

\section{Acknowledgements}

We wish to thank Professor B. Weiss for acquainting us with Körner's result and for his contribution to this paper.

\section{REFERENCES}

[FW] H. Furstenberg \& B. Weiss. The finite multipliers of infinite transformation. Lecture Notes in Maths 688 Springer: 1978, pp. 127-132.

[K] T. W. Körner. Recurrence without uniform recurrence. Ergod. Th. \& Dynam. Sys. 7 (1987), 559-566.

[F] H. Furstenberg. The structure of distal flows. Amer. J. Math. 85 (1963), 477-515.

[V] W. A. Veech. The equicontinuous structure relation for minimal abelian transformation groups. Amer. J. Math. 90 (1968), 723-732.

[P] K. E. Petersen. Disjointness and weak mixing of minimal sets. Proc. Amer. Math. Soc. 24 (1970), 278-280.

[Ke-R] H. B. Keynes \& J. B. Robertson. Eigenvalue theorems in topological transformation groups. Trans. Amer. Math. Soc. 139 (1969), 359-369.

[E-Ke] R. Ellis \& H. Keynes. A characterization of the equicontinuous structure relation. Trans. Amer. Math. Soc. 161 (1971), 171-183.

[B] I. V. Bronstein. Extensions of Minimal Transformation Groups. Susthoff and Noordhoff: Alphen aan rijn, 1979.

[C] J. Clay. Proximity relation in transformation groups. Trans. Amer. Math. Soc. 108 (1963), 88-96.

[E] R. Ellis. Distal transformation groups. Pac. J. Math. 8 (1958), 401-405.

[Ke] H. B. Keynes. Lifting of Topological entropy. Proc. Amer. Math. Soc.

[G-W] S. Glasner \& B. Weiss. On the construction of minimal skew products. Isl. J. Math. 34 (1979), 321-336. 